HCC and gastric cancer. It is more important that 61 gene mutations in the pancreatic cancer data set do not coincide with the other two datasets.

Among them, KRAS is located on the chromosome $12 \mathrm{P} 12.1$, gene type is a protein-encoding gene. The rate of mutation in pancreatic cancer is $137 / 163$ (84.05\%). The number of mutant species is 13 . The total survival rate of KRAS mutations in 162 cases of pancreatic cancer was significantly lower than that no mutation in KRAS $(p=1.18 \mathrm{e}-2)$. SCN5A is located on the chromosome $3 \mathrm{p} 22.2$, gene type is a proteinencoding gene. The rate of mutation in pancreatic cancer is $10 / 163(6.13 \%)$. The number of mutant species is 15 . The total survival rate of SCN5A mutations in 162 cases of pancreatic cancer was significantly lower than that no mutation in SCN5A $(p=2.99 \mathrm{e}-2)$.

Conclusions There is a significant correlation between the KRAS and SCN5A gene mutations and the prognosis of pancreatic cancer.

\section{IDDF2018-ABS-0101 RECURRENT GENE AMPLIFICATION SIGNATURE ASSOCIATED WITH INTRAHEPATIC METASTASIS OF HEPATOCELLULARCCARCINOMA}

${ }^{1}$ Manling Huang*, 'Sui Peng, ${ }^{2}$ Zongren Wang, 'Lixia Xu, ${ }^{3}$ Ming Kuang. 'Department of Gastroenterology, The First Affiliated Hospital of Sun Yat-sen University, Guangzhou, China; ${ }^{2}$ Department of Urology, The First Affiliated Hospital of Sun Yat-sen University, China; ${ }^{3}$ Department of Liver Surgery, The First Affiliated Hospital of Sun Yat-sen University, China

\subsection{6/gutjnl-2018-IDDFabstracts.48}

Background About $50 \%-75 \%$ of hepatocellular carcinoma (HCC) cases present with multiple intrahepatic foci in the liver at diagnosis, with poor prognosis and limited therapeutic options available. In this study, we aimed to identify recurrent genetic alterations of multifocal HCC.

Methods Whole genome sequencing and RNA sequencing were performed in 12 tumours and six matched non-tumour liver tissue samples to screen recurrent alterations in multifocal tumours. The selected genes were then analysed using data from The Cancer Genome Atlas (TCGA). Their biological functions were explored by in vitro experiments. Clinical impact of targeted genes was assessed in 60 patients with multifocal HCC in our medical centre.

Results ZNF687, ANXA9 and RABIF were identified as top candidates for their copy number gain and upregulated transcriptional activity in 10 tumours. The mRNA expression of these genes was upregulated in tumour tissues, with a positive correlation with gene amplification in 370 HCC cases from TCGA. Functional studies of ZNF687, ANXA9 and RABIF revealed that they could significantly increase cell proliferation and migration. Furthermore, the protein expression of ZNF687 was significantly higher in tumour tissues as compared with their adjacent normal tissues and overexpression of ZNF687 was significantly associated with shortened recurrence-free survival and overall survival in patients with multifocal HCC.

Conclusions We have identified three recurrent genetic alterations of multifocal HCC - the amplification and overexpression of ZNF687, ANXA9 and RABIF, which were highly associated with intrahepatic metastasis. Our findings may inspire innovative therapeutic approaches for multifocal HCC.

\section{IDDF2018-ABS-0132 IRREVERSIBLE ELECTROPORATION INDUCES IMMUNOGENIC CELL DEATH IN HEPATOCELLULAR CARCINOMA AND PROMOTES SYSTEMIC ANTI-TUMOUR IMMUNE EFFECTS IN MICE MODELS}

${ }^{1}$ Zihao Dai*, ${ }^{1}$ Junbin Liao, ${ }^{2}$ Sui Peng. 'Department of Liver Surgery, The First Affiliated Hospital of Sun Yat-sen University, China; ${ }^{2}$ Department of Gastroenterology and Hepatology, The First Affiliated Hospital of Sun Yat-sen University, China

\subsection{6/gutjnl-2018-IDDFabstracts.49}

Background The therapeutic efficacy of ablation on hepatocellular carcinoma (HCC) is impeded due to high tumour recurrence. The immune response induced by ablation has provided new insights into tumour monitoring and elimination. Irreversible electroporation (IRE) ablates tumours through high frequency electric pulse in a non-thermal manner, which could preserve more intact tumour antigens and provide a more powerful anti-tumour immune response theoretically. We aimed to explore the effects of immune reaction mediated by IRE.

Methods In vitro, characteristics of HCC cell death were determined by trypan blue staining, flow cytometrys (FCS) and transmission electron microscope analysis. Immunogenic cell death (ICD) was detected by calreticulin or HSP70 exposure by FCS, ATP secretion by luciferase and DC maturation assay. In vivo, C57BL/6 mice were employed to establish tumour ablation model, in which dynamics of immune infiltration were analysed by FCS and immunohistochemistry. The adaptive immune response was further confirmed by CD8 blockade, and vaccine experiments were performed.

Results We found that IRE ablation could effectively result in HCC cell death via necrosis and induced positive molecular determinants of ICD including increased ecto-CRT and ectoHSP70 exposure and elevated extracellular ATP level. In addition, the dendritic cells co-cultured with IRE-induced tumour lysis were activated evidenced by upexpression of co-stimulators and increased secretion of cytokine. Using mice model, we found IRE significantly inhibited tumour growth accompanied by increased CD $8+$ IFN $-\gamma+\mathrm{T}$ cells and reduced PD- 1 + cells and Treg. Depletion of CD8 $+\mathrm{T}$ cells abolished the therapeutic effect of IRE that local tumour recurrence and distant metastasis were promoted. IRE-based vaccine experiments showed vaccinated mice significantly resisted secondary tumour induction and demonstrated a long-term immunological memory response.

Conclusions Our study revealed that IRE treatment trigged ICD, enhanced immune response upon tumour recurrence and distant metastasis through CD8 $+\mathrm{T}$ cells and induced alleviation of immunosuppression.

\section{IDDF2018-ABS-0139 METALLOTHIONEIN 1G IS SILENCED BY DNA METHYLATION AND CONTRIBUTES TO THE PATHOGENESIS OF HEPATOCELLULAR CARCINOMA}

${ }^{1}$ Xiao-xing Li*, ${ }^{1} J u-d e n g$ Zeng, ${ }^{2}$ Ning Zhang, ${ }^{3} G u i-j u n$ Zhao, ${ }^{2}$ Li-xia Xu, ${ }^{1}$ Yang Yang, ${ }^{1}$ Xiaoyi Xu, ${ }^{1}$ Meng-ke Chen, ${ }^{1}$ Hui-yun Wang, ${ }^{1}$ Steven Xiao-feng Zheng. ${ }^{~}$ State Key Laboratory of Oncology in South China, Collaborative Innovation Center for Cancer Medicine, Sun Yat-Sen University Cancer Center, Guangzhou, Guangdong, China; '2Department of Gastroenterology, The First Affiliated Hospital, Sun Yat-sen University, Guangzhou, Guangdong, China; ${ }^{3}$ Department of Gastroenterology and Hepatology, Inner Mongolia People's Hospital, Hohhot, Inner Mongolia Autonomous Region, China 